\title{
Large size multi-gap resistive Micromegas for the ATLAS New Small Wheel at CERN
}

\author{
Dimitrios Fassouliotis 1,* \\ National and Kapodistrian University of Athens, \\ Panepistimioupolis, Athens, Greece \\ E-mail: Dimitrios.Fassouliotis@cern.ch
}

Large size multi-gap resistive strips Micromegas have been chosen together with the smallstrips TGC (sTGC) to be mounted on the New Small Wheel (NSW) upgrade of the ATLAS Muon Spectrometer. The NSW is the most ambitious and challenging upgrade of the ATLAS experiment for LHC-Phase1 (the current long shutdown) and will exploit its full capabilities after the Phase2 upgrade of LHC when the luminosity will reach values up to $7.5 \times 10^{34} \mathrm{~cm}^{-2} \mathrm{~s}^{-1}$ severely impacting on the ATLAS muon forward reconstruction and trigger. Four types of large size quadruplets Micromegas detectors are currently in series production in France, Germany, Italy, Russia and Greece. At CERN the integration of the modules and their assembly in sectors composing the wheel is well advanced. All the procedures of quality control are in place and steadily running. In this presentation the main challenges of the project will be reviewed. The achievement of the requirements for these detectors revealed to be even more challenging than expected, when scaling from the small prototypes to the large dimensions. We will describe the encountered problems, to a large extent common to other micro-pattern gaseous detectors, and the adopted solutions. In addition, the work of the integration of the detectors at CERN and the results from the validation tests at CERN on Micromegas sectors in their final configuration, will be also presented.

40th International Conference on High Energy physics - ICHEP2020

July 28 - August 6, 2020

Prague, Czech Republic (virtual meeting)

\footnotetext{
${ }^{1}$ On behalf of the ATLAS Muon Collaboration.

* Speaker
} 


\section{ATLAS New Small Wheel}

The ATLAS collaboration [1] has decided to substitute the Small Wheels of the end-cap muon spectrometer, with the New Small Wheels (NSW) [2] which will contain: Micromesh Gaseous Structure (Micromegas) primarily for precision tracking and Small strip Thin Gap Chambers (sTGC) primarily for trigger. NSW is the most ambitious and challenging Phase-I ATLAS upgrade. NSWs are designed to operate also at the high luminosity LHC (HL-LHC) era. The NSWs have a diameter of $10 \mathrm{~m}$ and they are positioned at a distance of $7 \mathrm{~m}$ along the beam direction, thus covering a pseudorapidity interval of $1.3<|\eta|<2.7$. The motivation of this upgrade is to reduce substantially the expected fake rate of the single level-1 muon trigger, without increasing the transverse momentum threshold and in parallel to maintain the excellent efficiency and resolution of the muon tracking at very high rates (reaching the value of $15 \mathrm{kHz} / \mathrm{cm}^{2}$ at HL-LHC operation). Each NSW consists of 16 active layers (8 layers of Micromegas and 8 layers of sTGC), with very high segmentation, allowing high redundancy for tracking and pattern recognition.

The NSW will preserve the geometry of the present SW. Each NSW consists of 8 small and 8 large sectors which are overlapping on their edges. Each sector consists of two Micromegas wedges, which are placed on an aluminum support (spacer frame) to make a double wedge (DW) and two sTGC wedges which are positioned on each side of the Micromegas DW. All the wedges consist of four active layers. The sectors are installed on a mechanical support which combines aluminium structures (the spokes) supporting the detectors and holding the alignment bars, with a steel disk (the JD) sitting from the interaction point side, used for radiation shielding and as return of the magnetic flux. The layout of the NSW is shown on figure 1(a) and the current real view of NSW side A with 6 small sectors installed on figure 1(b).

The operational principle of Micromegas is discussed in detail elsewhere [3], [4]. Very briefly, the general operation principle is the following. The gas volume is divided by a metallic mesh in two parts. The first one corresponds to the drift area where the primary ionization takes place. The second one is the region where the amplification avalanche takes place. The Micromegas used for the NSW are characterized by the use of a resistive anode to allow a better spark protection. The strip width is $300 \mu \mathrm{m}$ and the pitch $425-450 \mu \mathrm{m}$. The mesh is integrated in the drift panel structure and not coupled with the pillars. It is at ground potential. The baseline gas mixture consists of $93 \%$ $\mathrm{Ar}$ and $7 \% \mathrm{CO}_{2}$. In case of $\mathrm{ArCO}_{2}$ 93-7 gas mixture, the applied $\mathrm{HV}$ on the drift gap (width $5 \mathrm{~mm}$ ) and the amplification gap (width $128 \mu \mathrm{m}$ ), are $-300 \mathrm{~V}$ and $570 \mathrm{~V}$ respectively. The corresponding electric fields are $600 \mathrm{~V} / \mathrm{cm}$ and $45 \mathrm{kV} / \mathrm{cm}$ respectively.

\section{Construction of the Micromegas chambers}

Each Micromegas DW is composed by two wedges with four layers each. Each wedge comprises two eta layers for measuring the precision coordinate and two stereo layers with inclined strips $\left( \pm 1.5^{\circ}\right)$ to provide also the second coordinate. Each wedge is composed by two modules (or quadruplets) of type 1 or 2 as shown in figure 2(a). Thus, there are 128 modules in total of four different types, to be constructed to complete the two NSWs. The large type 1 modules (LM1) are constructed in France, LM2 in Greece and Russia, SM1 and SM2 in Italy and Germany, respectively. 


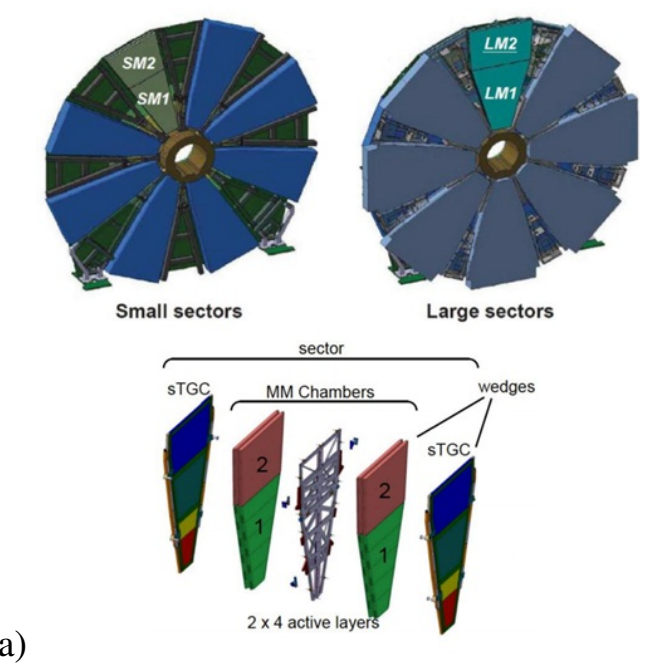

(b)

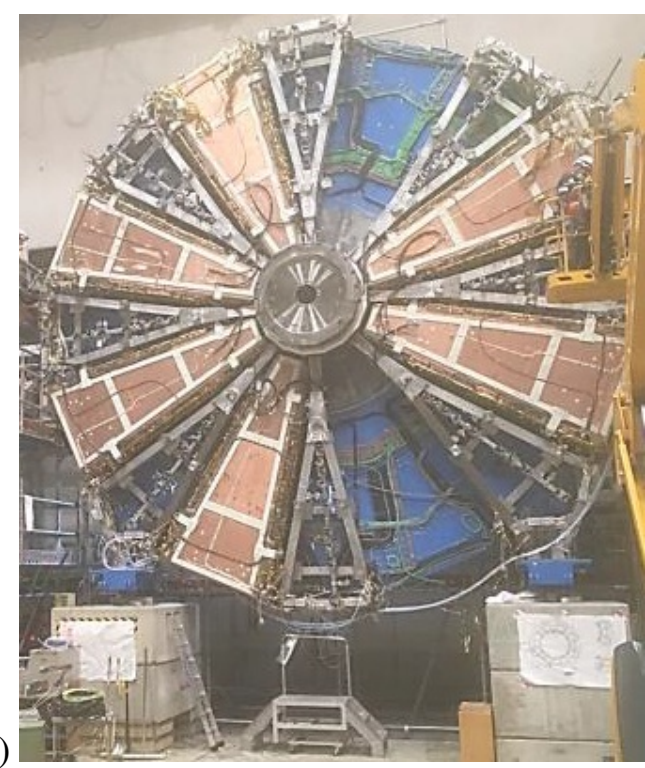

Figure 1: (a) Schematic of the layout of the NSW of ATLAS. Each wheel comprises 8 large and 8 small sectors and each sector two Micromegas and two sTGC wedges. (b) Photograph of the NSW side A as it appears on October 2020 with six small sectors installed. Copyright 2020 CERN for the benefit of the ATLAS Collaboration. CC-BY-4.0 license.

The surface of each Micromegas layer in a module ranges between 2 and $3 \mathrm{~m}^{2}$. Therefore the total area to be covered by Micromegas in NSW is $1200 \mathrm{~m}^{2}$.

The first step in the whole production procedure is the careful inspection of the industrial produced PCBs. This quality control procedure is performed on a specially equipped laboratory at CERN [5] and the validated PCBs are sent to the production sites. The construction of the modules involves several steps and requires high mechanical precision and very clean environment. As shown in figure 2(b), three drift panels, one of which is double sided, and two double sided read-out panels are used to construct a module. They are constructed in parallel following different production chains. The drift panels are assembled using granite tables in order to keep the planarity below the $20 \mu \mathrm{m}$ level. The meshes are glued and tensioned on an aluminium frame glued on the perimeter of the drift panel. The readout panels are assembled using a combination of granite tables and precision stiffback with an accuracy of $20 \mu \mathrm{m}$. The strips on both sides of the panel are aligned by a CCD reading a Rasnik mask and are glued together with the aluminum frame and the honeycomb. The drift panels and the readout panels are then assembled together into the final module. Before the final assembly the panels follow a strict cleaning protocol to remove any residual, dust or grease. The readout panels are brushed with both hard and soft brushes, using CIF as a detergent; they are then washed with demineralized water and dried in a ventilated oven at $40^{\circ} \mathrm{C}$. The mesh is polished to remove any imperfection or metallic remnants, then washed with hot water, brushed with a mixture of water and NGL and rinsed with demineralized water.

The final modules as well as the intermediate panels pass a very strict quality control procedure on the production sides before their transportation to CERN. For the final modules, the overall thickness, the planarity, the relative strip alignment and the gas tightness are measured. Their high 
(a)

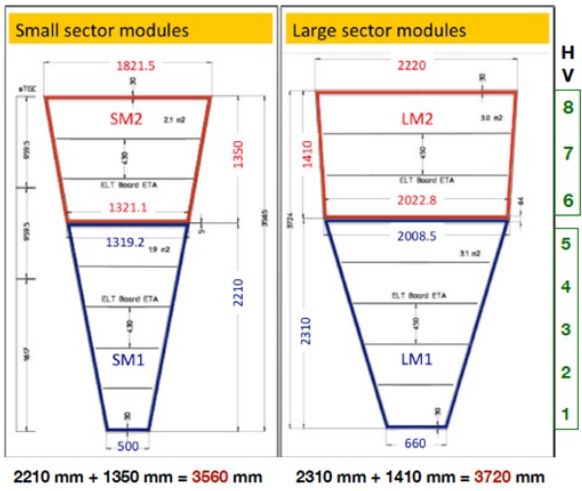

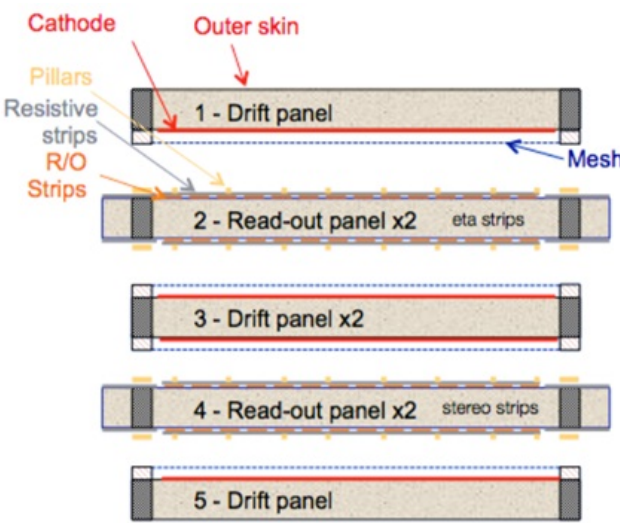

Figure 2: (a) The four different types of Micromegas modules. (b) Schematic representation of the construction of one Micromegas module. Copyright 2020 CERN for the benefit of the ATLAS Collaboration. CC-BY-4.0 license.

voltage stability, efficiency and gain homogeneity is measured with cosmic rays. The production of the Micromegas modules continues steadily. Already all the SM1 and most of the SM2 modules for both NSW sides have been produced.

\subsection{High voltage stability}

The Micromegas detectors scaling to large surfaces, the use of a floating mesh layout and a thin $128 \mu \mathrm{m}$ amplification gap, may give rise to HV instabilities, despite the resistive strip anode readout. These instabilities were observed in $10 \%$ of the HV sections of the initial modules that were produced. In parallel to the module production an investigation to understand and mitigate this effect was launched. Evidence of discharges happening close to the boundary of the active area of the detector was found. In certain HV sections (readout panels) detailed measurements have shown that the resistance was decreasing while approaching the boundaries of the active area, providing insufficient spark protection. The best procedure to stabilize the HV behavior was found to be the passivation of the low resistance areas close to the boundaries of the active area until a minimum value of the resistance of $0.8-1.0 \mathrm{M} \Omega$ is reached. The passivation of a region along the sides of the panels is performed through the deposit of a thin layer of araldite. Only a few modules were produced without passivation. All the rest have followed this procedure. More details on the passivation procedure can be found at [6].

In parallel, two other options to mitigate the HV instability issues are also investigated. One concerns the supply of HV with higher granularity than it was initially designed. Instead of supplying the whole layer of a module with one HV channel the possibility of supplying each individual readout panel ( 5 and 3 per layer for type 1 and type 2 modules respectively) with its own supply channel was studied. The results of this study are very encouraging as this design provides much higher flexibility to treat the unstable HV sections, thus increasing the overall efficiency of the detectors. The second option which is under study concerns the modification of the baseline gas mixture of the Micromegas by adding $2 \%$ isobutene. Although very encouraging results have been observed with this gas mixture [7], more long term studies are needed to validate this option. 


\section{Integration and commissioning of the Micromegas chambers}

The assembly, integration and commissioning of the Micromegas detectors take place at CERN in two experimental facilities in parallel. At building 899 (BB5) all modules arriving at CERN are validated, assembled on the spacer frame, provided with services and they are integrated with electronics. The complete Micromegas DW passes a series of quality assurance tests before it is transferred to building 191, where the combination with the sTGC wedges takes place, as well as the installation on the NSW. Since all the details concerning the integration part are presented at [8], this document will concentrate on the commissioning which is performed on the NSW. With the exception of the cosmic ray test [9], which presently is performed only at BB5, the validation procedures followed after the integration and at the commissioning stage are identical.

The fully equipped DW of Micromegas arrives at the building 191, where it is combined with two sTGC wedges to form a sector and it is installed on the NSW (presently side A). The installation procedure has already been performed eight times without facing any significant issues. Then, after the geometrical survey, the services are connected. This step includes the connection of the copper tubes to provide gas and cooling for the electronics, the temperature sensors to the DCS system, the low voltage and high voltage cables to the corresponding power supplies and the read-out fibers to the corresponding small boxes on NSW.

The commissioning procedure essentially contains two streams most parts of which can be performed in parallel. The first one concerns the gas tightness, the provision of gas flow, the control of the relative humidity inside the sector and finally the HV performance validation in terms of the spark rate. For all the small sectors integrated at BB5, 90\% of the HV sections are configured to work at the nominal $\mathrm{HV}$ value, $9 \%$ are set to lower value and $1 \%$ is disconnected. After the commissioning of three sectors on NSW at building 191, one additional read-out HV section out of 384 was found to be misbehaving and is currently under investigation. On the contrary, two unexpected issues were observed on the HV distribution of the drift side, but they were understood and fixed. The gas tightness of all the sectors commissioned so far is excellent.

The second stream of the commissioning, concerns the validation of the cooling system, the correct configuration and reading of the read-out electronics as well as the evaluation of the electronic noise conditions. Besides certain improvements that currently take place, the cooling system performance proved to be very stable allowing the electronic boards to work continuously without overheating. The DCS systems to control and monitor the electronics are fully functional and almost at their final condition and the DAQ procedures are advanced enough to fulfill all the commissioning tests. Several types of runs are recorded to characterize each of the 65536 electronic channels for each Micromegas sector. First the baselines are recorded to evaluate the electronic noise levels. Then, thresholds are set for each electronic channel and subsequently random trigger runs are performed using these thresholds to validate them. Pulse runs are used for the calibration of the read-out channels and the validation of the trigger path. Finally, the high rate communication electronics which will be used at the HL-LHC are also validated. Figure 3 shows two examples of the commissioning data taking. Figure 3(a) shows the thresholds set on the 512 electronic channels of one of the 128 frond-end boards. The homogeneity of the threshold values can be observed. Figure 3(b) shows the results of the connectivity test performed to validate the trigger path on one sector, where excellent response of all the electronic components can be observed. Using 
(a)

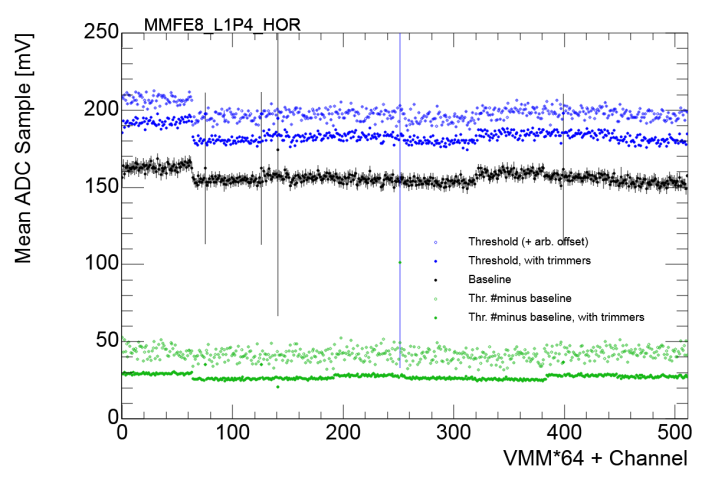

(b)

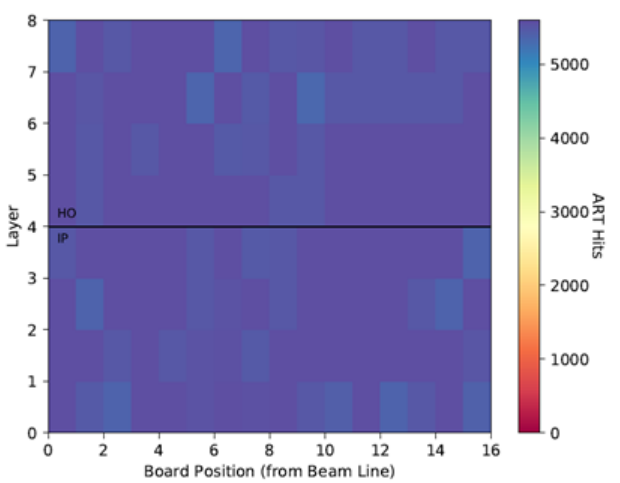

Figure 3: (a) Example of thresholds set for the 512 electronic channels of one frond-end board (b) The result of the trigger connectivity validation in one sector. Copyright 2020 CERN for the benefit of the ATLAS Collaboration. CC-BY-4.0 license.

the available infrastructure at building 191, the performance of the electronics of two sectors can be tested in parallel. The whole commissioning procedure has been applied up to now on three Micromegas sectors. While there is still place for optimization, several details have been tuned and a lot of experience have been accumulated on solving in situ several minor issues that may appear after the sectors' installation.

\section{References}

[1] ATLAS Collaboration, The ATLAS Experiment at the CERN Large Hadron Collider, JINST 3 (2008) S08003.

[2] ATLAS Collaboration, ATLAS New Small Wheel Technical Design Report, CERN-LHCC2013-006 ATLAS-TDR-020-2013 (2013).

[3] I. Giomataris et al., Micromegas in a bulk, NIM A 560 (2006) 405.

[4] T. Alexopoulos et al., A spark-resistant bulk-Micromegas chamber for high-rate applications, NIM A 640 (2011) 110.

[5] L. Longo, Production and Test of Micromegas boards for the ATLAS New Small Wheel project, these proceedings.

[6] I. Gnesi, The Micromegas chambers for the ATLAS New Small Wheel upgrade, JINST 15 C09019 (2020).

[7] L. Pezzotti, Irradiation and Gas studies of production chambers for the ATLAS-NSW MM, these proceedings.

[8] T. Vafeiadis, Integration and commissioning of ATLAS New Small Wheel Micromegas detectors with electronics at CERN, these proceedings.

[9] G. Rabanal Bolanos, Cosmic results with the final Micromegas sectors for the ATLAS Muon upgrade, these proceedings. 